Background Rugby participation rates are rising, particularly in the female game where a $60 \%$ increase in player numbers was observed from 2013 to 2017. Despite the recent growth, the female amateur game is lacking comprehensive long-term injury surveillance.

Objective To compare injuries in male and female amateur Rugby Union.

Design Prospective cohort study.

Setting Irish amateur clubs, during seasons 2017/18 ( $\mathrm{n}=15$ male clubs, 4 female) and 2018/19 ( $n=25$ male clubs, 7 female).

Participants Male $(n=958)$ and female $(n=234)$ amateur players.

Independent Variables Match exposure.

Main Outcome Measurements Match injury incidence and severity.

Results Overall incidence rates were 47.7 and 35.4/1,000 player hours for males and females respectively. Similarities existed between males and females regarding common diagnoses and injury occurrence, with 58\% of injuries occurring during the tackle. Concussion and ankle lateral ligament injuries were the most common diagnoses for both males (5.5 and 4.1/1,000 player hours) and females (5.5 and 3.9/1,000 player hours). However differences showed females suffering more injuries in the ruck compared to males (6.1 vs $3.8 / 1,000$ player hours) while males sustained more non-contact injuries compared to females (4.7 vs $1.4 / 1,000$ player hours). Females showed an earlier injury occurrence in the $2^{\text {nd }}$ quarter (9.4/ 1,000 player hours) plateauing into the $3^{\text {rd }}$ and $4^{\text {th }}$ quarters, whereas males had a $3^{\text {rd }}$ quarter injury peak $(15.2 / 1,000$ player hours). Concussion had the highest injury burden in males (190days/1,000 player hours), while anterior cruciate ligament injuries had the highest burden (307days/1,000 player hours) in females.

Conclusions Long-term prospective injury surveillance is vital to inform targeted prevention strategies. The earlier occurrence of injury in females should be investigated further to determine whether player substitution strategies may decrease injuries. Prevention strategies incorporating neuromuscular training should be considered, given the high rate of ankle ligament injuries in both males and females, and the burden of knee ligament injuries in females.

\section{EFFECTS OF A STRENGTH AND PROPRIOCEPTIVE TRAINING PROGRAMME ON NECK FUNCTION AND CONCUSSION INJURY RISK IN ELITE SCOTTISH RUGBY UNION PLAYERS}

${ }^{1}$ Stuart Bailey, 'Russell Martindale, ${ }^{2}$ Lars Engebretsen, ${ }^{3}$ Jen Sweeting, ${ }^{3}$ Jared Deacon, ${ }^{3}$ Florence Laing, ${ }^{3}$ Chris Leck, ${ }^{1,4}$ Debbie Palmer. ${ }^{1}$ School of Applied Sciences, Edinburgh Napier University, Edinburgh, UK; ${ }^{2}$ Oslo Sports Trauma Research Center, Oslo, Norway; ${ }^{3}$ Scottish Rugby Union, Edinburgh, UK; ${ }^{4}$ Institute for Sport, PE and Health Sciences, Moray House School of Education and Sport, Edinburgh, UK

\subsection{6/bjsports-2021-IOC.9}

Background Cervical musculature function (strength, endurance, and proprioception) may be a modifiable risk factor for concussion in contact sports. The ability to enhance neck function and reduce concussive risk needs to be determined.

Objective To assess the efficacy of a training programme to enhance neck function and lower match concussion injury risk in rugby union players.
Design Repeated-measures intervention with control.

Setting Premiership rugby union players in Scotland during the 2018/19 season.

Patients (or Participants) Premiership players selected for Scotland Rugby academies (intervention group; INT; $\mathrm{n}=30$ ) or those with their clubs (control group; CON; $n=20$ ).

Interventions (or Assessment of Risk Factors) A neck training programme was implemented twice per week during the 2018/19 season for INT, while CON performed no systematic neck training.

Main Outcome Measurements For both INT and CON neck strength (maximal voluntary contraction; MVC), endurance (exercise capacity; TTF) and proprioception pre- and post-season, and match concussion injury incidence were recorded.

Results Left and right cervical flexion MVC force and flexion TTF all significantly increased in the intervention group $(p<0.001)$, with no significant change in the control group. While there were increases in cervical flexion and extension MVC force in both groups from pre to post-season, there was a significantly greater increase amongst the intervention group $(p<0.05)$. Concussion incidence was lower in INT versus CON (INT: 7.7/1000 match hours; CON: $18.4 / 1000$ match hours). However, this was not a significant alteration in risk (incidence rate ratio: $0.42 ; 95 \%$ CI: 0.08-2.1).

Conclusions The neck function programme increased cervical MVC force and flexion exercise capacity, beyond any changes induced by a season of rugby union. The intervention group also had a lower incidence of concussion across the season. This pilot study shows good promise and highlights the need for further investigation.

\section{COMPARISON OF INJURIES AND ILLNESSES BETWEEN REGULAR COMPETITION AND SHORT-TERM MATCH CONGESTION DURING A FULL SEASON IN ELITE MALE PROFESSIONAL BASKETBALL}

\footnotetext{
1,2Steven Doeven, ${ }^{1}$ Michel S Brink, 'Barbara Huijgen, ${ }^{2}$ Johan de Jong, ${ }^{1}$ Koen Lemmink. ${ }^{1}$ Center for Human Movement Sciences, University of Groningen, University Medical Center Groningen, the Netherlands, Groningen, Netherlands; ${ }^{2}$ School of Sport Studies, Hanze University of Applied Sciences, the Netherlands, Groningen, Netherlands
}

\subsection{6/bjsports-2021-IOC.10}

Background It is crucial to balance load and recovery during short-term match congestion in basketball. Currently, it is unknown if higher total load during short-term match congestion lead to higher injury and illness rates.

Objective Aim of this study was to compare injuries and illnesses and total weekly load during 1-match weeks compared to $\geq 2$-match weeks in basketball.

Design During this prospective observational study, players were monitored during a full season.

Setting Two basketball teams participating in the domesticleague championship, CUP matches and Euro league were followed.

Patients (or Participants) Sixteen elite male professional basketball players participated in this study. Characteristics of the players were $($ mean $\pm S D)$ : age $24.8 \pm 2.0$ years, height 195.8 $\pm 7.5 \mathrm{~cm}$, weight $94.8 \pm 14.0 \mathrm{~kg}$, body fat $11.9 \pm 5.0 \%$ and $\mathrm{VO}_{2 \max } 51.9 \pm 5.3 \mathrm{~mL} \cdot \mathrm{kg}^{-1} \cdot \mathrm{min}^{-1}$. 
Interventions (or Assessment of Risk Factors) In total 47 matches by basketball team A ( 9 players) and 41 matches by team B (7 players) were performed throughout the season. All training sessions and matches were executed as prescribed by the training and coaching staff without interference or manipulation.

Main Outcome Measurements The Oslo Sports Trauma Research Center (OSTRC) Questionnaire on Health Problems was used to collect data on injuries and illnesses on a weekly base. Furthermore, players filled in s-RPE and duration for each training and match. Prevalence's, severity scores, timeloss and total weekly load were compared for 1-match weeks and $\geq 2$-match weeks. The data were analyzed using multilevel modeling.

Results Prevalence of injuries and illnesses were $18.1 \%$ and 4.6\% for 1-match weeks and $17.2 \%$ and $3.3 \%$ for $\geq 2$-match weeks. Severity scores and time-loss were not significantly different for 1-match weeks compared to $\geq 2$-match weeks. Total weekly load was lower during $\geq 2$-match weeks compared to 1-match weeks.

Conclusions No significant differences for injuries and illnesses were observed between 1 -match weeks and $\geq 2$-match weeks. Coaches appeared to reduce training load to compensate for multiple matches during short-term match congestion.

\section{EVALUATION OF IN-EAR SENSOR SYSTEMS FOR QUANTIFYING HEAD IMPACTS IN YOUTH FOOTBALL}

\begin{abstract}
${ }^{1,2}$ Stian Bahr Sandmo, ${ }^{3,4,5}$ Andrew S Mclntosh, ${ }^{1}$ Thor Einar Andersen, ${ }^{6,7}$ Inga K Koerte, ${ }^{1}$ Roald Bahr. 'Oslo Sports Trauma Research Center, Norwegian School of Sport Sciences, Oslo, Norway; ${ }^{2}$ Faculty of Medicine, University of Oslo, Oslo, Norway; ${ }^{3}$ School of Engineering and ACRISP, Edith Cowan University, Joondalup, Australia; ${ }^{4}$ Monash University of Accident Research Centre, Monash University, Melbourne, Australia; ${ }^{5}$ Mclntosh Consultancy and Research, Sydney, Australia; ${ }^{6}$ Department of Child and Adolescent Psychiatry, Psychosomatic, and Psychotherapy, Ludwig-Maximilian-University, Munich, Germany; 'Department of Psychiatry, Psychiatry Neuroimaging Laboratory, Brigham and Women's Hospital, Harvard Medical School, Massachusets, USA
\end{abstract}

\subsection{6/bjsports-2021-IOC.11}

Background Wearable sensor systems have the potential to quantify head kinematic responses of head impacts in football. However, on-field use of sensors (e.g. accelerometers) remains challenging due to factors such as poor coupling to the head.

Objective To test the validity of a novel in-ear sensor for quantifying head-impact exposure in youth football.

Design Descriptive laboratory study/validation study.

Setting Youth football.

Participants Six male youth football players (15.3 \pm 0.3 years). Evaluations In step 1, the sensor was mounted to a Hybrid III headform (HIII) and impacted with a linear impactor or football (range: 9-144g). Accelerative forces, including peak linear acceleration (PLA), were obtained from both systems. In step 2 , six youth soccer players wore sensors during a structured training protocol including heading and non-heading exercises; in step 3, they completed two regular football sessions. For each recorded accelerative event, PLA outputs were compared to video.

Main Outcome Measurements In step 1, random and systematic error were calculated using HIII as reference. In steps 2 and 3 , mean values $( \pm S D)$ were calculated for $(1)$ all heading and (2) all non-heading events. Receiver operating characteristic curves were used to determine the sensor's discriminatory capacity in both on-field settings, and cut-off values for predicting outcomes were identified.

Results In step 1, random and systematic error were both $11 \%$ for PLA. In step 2, heading events resulted in higher absolute values (PLA $=15.6 \pm 11.8 \mathrm{~g})$ than non-heading events $(\mathrm{PLA}=4.6 \pm 1.2 \mathrm{~g})$; area under the curve (AUC) was 0.98. In step 3, AUC was $>0.99$. A 9g cut-off value yielded a positive predictive value of $100 \%$ in the structured training protocol vs. $65 \%$ in regular football sessions.

Conclusions The in-ear sensor displayed considerable random error and overestimated head impact exposures substantially. It showed excellent on-field accuracy for discriminating headings from other accelerative events, but secondary means of verifying events are still necessary.

\section{HEAD IMPACT EXPOSURE IN YOUTH FOOTBALL - ARE CURRENT INTERVENTIONS HITTING THE TARGET?}

${ }^{1,2}$ Stian Bahr Sandmo, ${ }^{1}$ Thor Einar Andersen, 3,4 Inga K Koerte, ${ }^{1}$ Roald Bahr. 'Oslo Sports Trauma Research Center, Norwegian School of Sport Sciences, Oslo, Norway; ${ }^{2}$ Faculty of Medicine, University of Oslo, Oslo, Norway; ${ }^{3}$ Department of Child and Adolescent Psychiatry, Psychosomatic, and Psychotherapy, Ludwig-Maximilian-University, Munich, Germany; ${ }^{4}$ Department of Psychiatry, Psychiatry Neuroimaging Laboratory, Brigham and Women's Hospital, Harvard Medical School, Massachusets, USA

\subsection{6/bjsports-2021-IOC.12}

Background Restrictions on heading in youth football have been implemented in the U.S. to limit head-impact exposure up until the age of 13 . However, current interventions remain poorly guided by evidence, and providing more accurate data on heading exposure is key to assess risk.

Objective To quantify heading exposure in youth football, assessing the effects of sex and age.

Design Prospective cohort study, based on direct observation of a convenience sample of football matches played during an international youth football tournament. The tournament was played without heading restrictions, with separate sex and age groups.

Setting Youth football.

Participants Male and female teams with players aged 11-19 years. A total of 267 matches was observed.

Independent Variables Sex and age. The elite senior level was included for comparison, using video analysis.

Main Outcome Measurements All heading events were registered, classified and assigned to individual players. Heading rates were calculated for each sex and age group.

Results We observed a total of 4011 player hours (1927 player hours for females, 2083 player hours for males). Males headed more frequently than females (2.7 vs. 1.8 headers/ player hour; $\mathrm{p}<0.001)$. Heading rates increased with age (ANOVA, p<0.001), approaching the elite senior level for players 16 years and older. There was substantial variation within teams for all age and sex groups, with the widest range (1-18 headers) observed for girls aged 19. Girls younger than 12 years had the lowest exposure, with an average of less than two players per team heading the ball, each with 1-2 headers.

Conclusions Age and sex influence head-impact exposure in youth football, and warrants careful consideration when 